\title{
DIAGNOSTIC VALUE OF SERUM LACTATE DEHYDROGENASE ISOENZYME AND AMINO ACID PATTERNS IN SEVERAL SCHISTOSOMAL AND NON-SCHISTOSOMAL DISORDERS AS COMPARED TO OTHER BIOCHEMICAL PARAMETERS
}

\author{
SAMIA A. AHMED* AND MOHAMED Z. GAD \\ *Department of Medicinal Chemistry, National Research Center and \\ ${ }^{\dagger}$ Biochemistry Department, Faculty of Pharmacy, Cairo University, Cairo, Egypt
}

\section{SUMMARY}

\begin{abstract}
Serum lactate dehydrogenase ( $\mathrm{LDH}$ ) isoenzyme and amino acid (a.a) patterns were evaluated in comparison to several other biochemical parameters for liver and renal function with the objective of clarifying the differential diagnosis of hepatic disorders and predicting the outcome of schistosomal infection in Egyptian patients. Patients examined included those with complicated hepatic disorders and others with different stages of schistosomal infestation, hepatoma or bladder cancer, in addition to a normal control group.

Several biochemical parameters appeared to be useful in establishing consistent differences or similarities between the studied groups. Examples are; elevated serum AST/ALT ratio and methionine content in chronic schistosomiasis, elevated serum urea/creatinine ratio and leucine content in all schistosomal patients and extremely high levels of $\mathrm{N}$-acetyl- $\beta$-D-glucosaminidase (NAG) in the urine of non-schistosomal bladder cancer patients. In addition, characteristic LDH isoenzyme profiles distinguish between the studied groups, in particular separating chronic schistosomiasis from schistosomal bladder cancer and hepatoma from other hepatic disorders.
\end{abstract}

KEY WORDS Lactate dehydrogenase isoenzymes Plasma amino acids Schistosomiasis Bladder cancer Hepatic and renal function tests

\section{INTRODUCTION}

Hepatoma and bladder carcinoma are both among the most important health problems in Egypt. Prognosis in hepatoma is grave and patients seldom survive for more than six months from the onset of symptoms (Michael, 1990). Many patients present when the tumor is already too advanced for surgery. The high incidence of bladder carcinoma in schistosomal patients also represents a great risk to Egyptian society where schistosomiasis remains endemic.

The importance of early diagnosis of cancer, specifically at the stage of progression of schistosomiasis to bladder cancer, or prior to the development of hepatoma, and the problems of differentiating between various cancer categories e.g schistosomal bladder cancer (SBC) vs non-schistosomal bladder cancer (NSBC) emphasise the need to search

Address for Correspondence: Dr. Mohamed Z. Gad, Biochemistry Department, Faculty of Pharmacy, Cairo University, Kasr ElAini st., Cairo, Egypt. Tel: (202) 3639307; Fax: (202) 3624105 
for new biochemical parameters or tumor markers. Despite considerable progress, we still lack an "ideal" tumor marker, especially in SBC. Furthermore, the radiological and pathological parameters upon which treatment is based, are relatively crude. (Pohl, 1992).

Liver and kidney function tests have repeatedly been shown to reflect the biological behaviour of different malignant tumors (Huang et al., 1988). However, these are, in the main, non-specific and cannot differentiate between different types of liver or urinary tract disorders or the different stages of cancer. In the present study, we have evaluated serum LDH isoenzyme and a.a patterns in patients with different schistosomal and nonschistosomal liver disorders and bladder malignancies.

\section{MATERIALS AND METHODS}

\section{Patient selection and classification}

All investigated cases were adult Egyptian patients presenting either to the National Cancer Institute (NCI), Cairo University (cancer patients) or to Theodor Bilharz Institute (TBI), Cairo (hepatic and schistosomal patients). The patients were categorized as follows:

Group I (G I): 10 normal control subjects with no evidence of parasitic infection, liver, kidney or neoplastic diseases.

Group II (G II): 7 patients with hepatic cirrhosis accompanied by splenomegaly and ascites.

Group III (G III): 7 patients with hepatic cirrhosis splenomegaly and hepatocellular carcinoma.

Group IV (G IV): 9 patients with schistosoma hematobium infection complicated with viral hepatitis and hepatic cirrhosis.

Group V( G V): 6 patients with schistosoma hematobium complicated with hepatic fibrosis.

Group VI (G VI): 6 patients with active schistosoma hematobium infection but no associated complications.

Group VII (G VII): 6 patients with hepatocellular carcinoma but without cirrhosis.

Group VIII (G VIII): 6 patients having non-schistosomal bladder carcinoma (NSBC).

Group IX (G IX): 6 patients with bladder carcinoma secondary to schistosoma hematobium infection (SBC).

Group X $(\mathrm{G} \mathrm{X})$ : 6 patients with chronic schistosoma hematobium infection. For this group, only the serum a.a pattern was examined.

A thorough clinical examination was undertaken, with complete haemogram, urine analysis and stool analysis for detection of ova and parasites. None of the patients had overt clinical heart disease, symptomatic myopathy, nephrosis or pancreatitis.

\section{Biochemical parameters}

The following were undertaken for all patients and controls

1) Hepatic function tests: Serum total bilirubin (Jendrassik and Grof, 1938), Alanine aminotransferase, ALT (Wrobklewski and La Due, 1956), Aspartate aminotransferase, AST (Wilkinson, 1976), Alkaline phosphatase, ALP (Tietz and Rinker, 1983) and plasma total protein (Lowry et al., 1951). 
2) Renal function tests: Serum creatinine (Henny et al., 1985) and urea (Fawcett and Scott, 1960)

3) Serum uric acid: (Watts 1974)

4) Serum lactate dehydrogenase activity: (Wacker et al., 1956)

5) Urinary $\mathrm{N}$-acetyl-ß-D-glucosaminidase: adapted from Beaufay et al., 1974.

6) Serum amino acid pattern: briefly, serum samples were deproteinized by mixing with 1 volume of sulfosalicylic acid (Hamilton, 1962). The precipitate was discarded after centrifugation, and the a.a were evaluated in the supernatent with a Beckman $121 \mathrm{CL}$ amino acid analyzer

7) LDH isoenzyme pattern: One patient from each group was selected for the study. Serum samples were exposed to electrophoretic separation of LDH isoenzymes in a buffered agarose gel using Beckman Paragon lactate dehydrogenase isoenzyme electrophoresis kit.

\section{Statistical analysis}

Results were analyzed by student " $t$ " test, $p$ values of 0.05 or less were considered significant.

\section{RESULTS}

\section{Effects on liver function tests}

Results shown in Figure 1 indicated a mild but significant increase in plasma total protein in several test groups. The highest increase was in G III (cirrhosis, splenomegaly and hepatoma). In contrast, a significant decrease in plasma total protein was observed only in G V schistosomiasis complicated with hepatic fibrosis. However, despite the significant difference from normal control values observed for most groups, all results were still around normal ranges for plasma proteins and the findings were of limited diagnostic value.

Serum total bilirubin was also elevated for several groups. The highest increase was noticed for groups III, IV and VII, all of whom had complicated hepatic disorders, but a significant increase was apparent for all schistosoma infected groups. For alkaline phosphatase, a noticeable increase in the enzyme activity was observed in all groups except VII (hepatoma). Maximum elevation - about twice the control value — was observed in hepatitis (G IV). However, the range of results was narrow. In contrast, more distinctive changes were found for serum ALT and AST. For both enzymes, activity was significantly elevated in all studied groups. However, groups associated with chronic schistosomiasis (IV, V and IX) showed not only the highest level of both enzymes but also the highest AST/ALT ratios.

\section{Effects on renal function tests}

Despite the significant elevation of serum creatinine observed in all groups except VIII (Figure 1), increments did not exceed twice the control level. Levels of serum creatinine in SBC patients (IX) were higher than in NSBC patients (VIII). For serum urea, a bimodal pattern was observed with reductions in groups II, III and VII and elevation in groups IV, VI, IX, maximal in group V. Consequently, the ratio of serum urea/serum creatinine was variable from one group to another being relatively low for groups II and III and high in groups V and IX. 



Figure 1. Changes in Biochemical parameters in different hepatic and bladder disorders. Groups and number of samples are indicated in the text. Results are mean + SD. Units are mg/dl (uric acid, urea), mg/l (bilirubin, creatinine), g/dl (total protein), U/l (ALP, AST, ALT).

* indicates significant difference from normal at $\mathrm{p} \leq 0.05$. 
No obvious modulation of serum uric acid was apparent in the results. While significant decreases from control values were found in groups II and III and increases for groups IV and V, mean levels for all groups remained within (or close to) the normal physiological range $(3-7 \mathrm{mg} / \mathrm{dl})$.

\section{Effects on urinary $N$-acetyl- $\beta$-D-glucosaminidase activity}

Results shown in Figure 2 indicate a large difference between the mean NAG activity of NSBC patients and the mean for all other groups, including normal controls. No significant difference from mean control activity was observed for either the active schistosomiasis or SBC patients.

\section{Modulations of amino acid pattern}

Figure 3 represents the serum amino acid pattern of five different groups as compared to the a.a profile of normal controls. Mild elevation of the total content of a.a was shown for all groups, reaching 30\% above control level in chronic schistosomal patients. The most characteristic and reproducible change in all groups is the decrease in glutamine content which was only $57,41,45,63$ and $53 \%$ of control level for patients in groups NSBC, active schistosomiasis, SBC, chronic schistosomiasis and hepatoma respectively. Comparing the data for individual groups, it is noticeable that marked elevation of leucine, acidic a.a (Glu and Asp) and arginine content occurs in active bilharziasis patients. In the chronic schistosomiasis group these specific elevations were maintained and accompanied by a characteristic and unique three fold increase in the methionine level compared with all other groups. This finding applied to all six patients in group X. No other characteristic pattern of change distinguishes the serum a.a pattern of chronic bilharziasis from that of SBC patients. On the other hand, SBC patients differed from the NSBC patients in the contents of basic a.a (Arg, His, Lys and Orn). All schistosomal patients whether active, chronic or with bladder cancer, displayed a characteristic increase in the leucine content. Several criteria distinguish hepatoma patients from other groups, including highest level of glutamic acid and lowest content of leucine and threonine.

\section{Differences in LDH isoenzyme profiles:}

Levels of serum LDH were significantly elevated in all groups except II and V (Table 1). Maximum increase was found in the hepatitis group (IV) followed by hepatoma patients (VII). Active schistosomiasis patients (VI) generally showed higher levels than those with chronic infestation (V).

Only one patient from each group has been studied for LDH iso-enzyme pattern. Results are therefore preliminary. They are, nevertheless, striking (Table 1) with overall LDH levels generally showing an increase but the distribution of activities between the five iso-enzymes showing much greater deviations from control values.

The normal controls exhibited three major peaks; LD1, LD2 and LD3 representing $34.4,28.2$ and $23.2 \%$ of total LDH activity respectively plus two smaller peaks LD4 and LD5 accounting for $7.1 \%$. This pattern was remarkably reversed in the active schistosomiasis patient (VI) where LD4 and LD5 became major peaks representing 41.2 and $18.3 \%$ of total LDH respectively. Development of chronic schistosomiasis with fibrosis (V) did not markedly change the active schistosomiasis LDH pattern other than a mild increase in LD1 from 13.6 to $22.1 \%$ and a further decrease in LD2 from 8.2 to $2.1 \%$. 


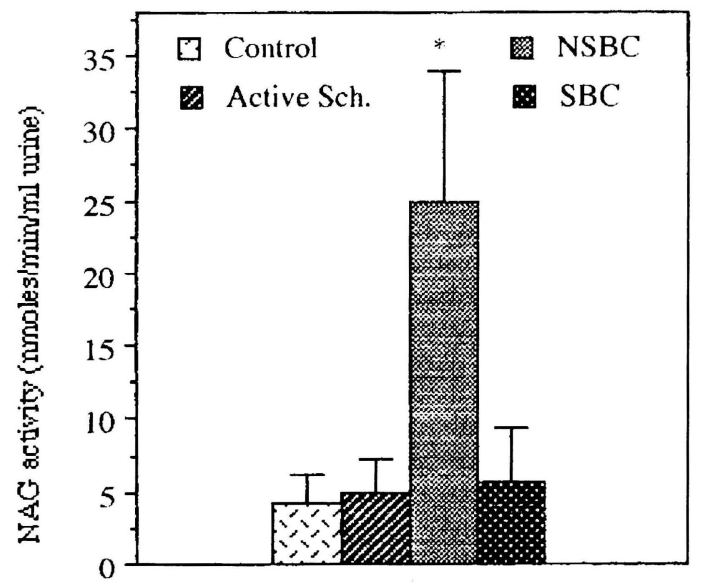

Figure 2. Changes in NAG activity in the urine of schistosomal patients. Results are mean \pm SD. Groups are normal control ( $\mathrm{N}=11$ ), active schistosomiasis (active $\mathrm{Sch}$., $\mathrm{N}=7$ ), schistosomal bladder cancer $(\mathrm{SBC}, \mathrm{N}=6$ ), non-schistosomal bladder cancer $(\mathrm{NSBC}, \mathrm{N}=6)$. Units are nmoles $/ \mathrm{min} / \mathrm{ml}$ urine.

* indicates significant difference from normal at $\mathrm{p} \leq 0.05$.

Table 1. LDH isoenzymes profile of patient groups as compared to normal control. Groups and number of samples are indicated in the text. "Total" represents serum LDH concentration $\pm \mathrm{SD}$.

\begin{tabular}{lccccccc}
\hline G I & 34.4 & 28.2 & 23.2 & 7.1 & 7.1 & $140 \pm 14$ \\
G II & 25.5 & 4.0 & 15.6 & 34.9 & 20.0 & $160 \pm 29$ \\
G III & 23.2 & 1.6 & 17.1 & 40.2 & 17.9 & $195^{*} \pm 11$ \\
G IV & 25.1 & 8.7 & 23.5 & 30.8 & 11.9 & $424 * \pm 39$ \\
G V & 22.1 & 2.1 & 15.1 & 41.0 & 19.7 & $171 \pm 233$ \\
G VI & 13.6 & 8.2 & 18.7 & 41.2 & 18.3 & $234^{*} \pm 17$ \\
G VII & 16.7 & 20.4 & 19.3 & 14.1 & 29.5 & $332 * \pm 22$ \\
G VIII & 5.7 & 8.3 & 29.6 & 36.5 & 19.9 & $182^{*} \pm 8$ \\
G IX & 27.6 & 21.4 & 28.2 & 11.7 & 11.1 & $273^{*} \pm 13$
\end{tabular}



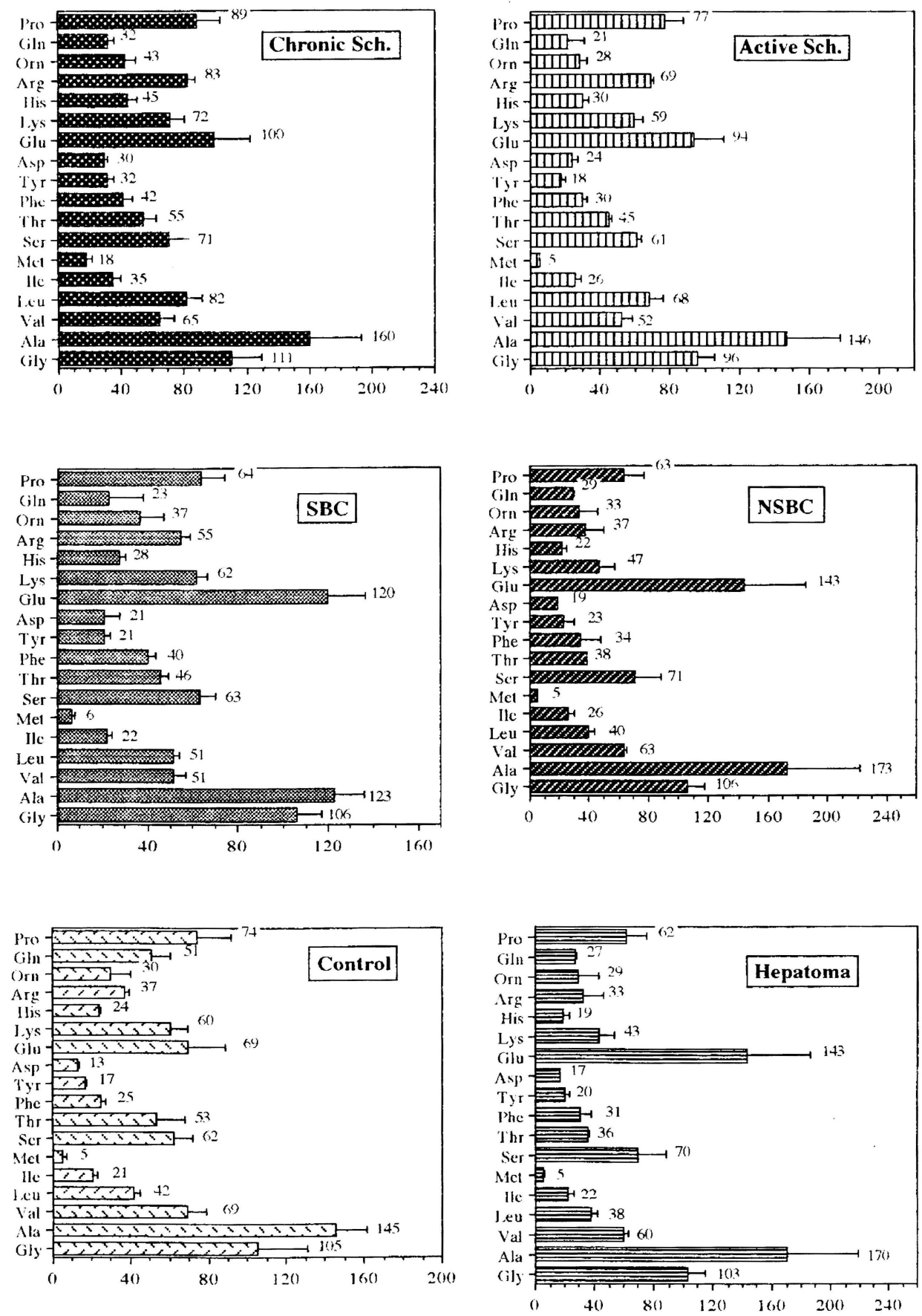

Figure 3. Serum amino acid pattern in five schistosomal and carcinoma disorders as compared to normal control. Results are mean \pm SD. Units are $\mu \mathrm{mol} / \mathrm{l}$. Description of the groups and number of samples are indicated in the text. Total concentration of serum a.a are: normal control: 912.9, Active Sch.: 974.5, Chronic sch.: 1194.8, SBC: 959.0, NSBC: 989.6, Hepatoma: 944.5 . 
This profile changed in the presence of viral hepatitis (IV) where the LD3 peak was increased at the expense of LD4 and LD5. Unlike the active schistosomiasis patient, the pattern in SBC patient (IX) was very similar to the normal control with no major peak for LD4. On the other hand, the difference between LDH isoenzyme pattern for NSBC and SBC were very conspicuous; with high activity in LD3, LD4 and LD5 for the former. The hepatoma patient (VII), exhibited a major LD5 peak. Complication of hepatoma with liver cirrhosis and splenomegaly (III) completely altered this pattern. For example LD1/LD2 ratio changed from 0.82 in hepatoma to 14.5 in the aforementioned patient. This pattern was comparable to that of groups II and IV who also suffered from chronic liver cirrhosis.

\section{DISCUSSION}

A generalized disturbance in liver function was obvious for all studied groups as indicated by all liver function tests including ALT, AST, ALP and total bilirubin. In schistosomoasis a diminished excretory function of liver has been demonstrated previously (Ragheb, 1956). Serum ALT and AST have been shown to be highly elevated in several mixed liver disorders in Egyptians (Mahmoud and Abdel Wahab, 1990). However, our data illustrate several new points. First, levels of total plasma proteins were not markedly altered in any of the studied groups. Second, different measures of liver function were not closely correlated with each other. For example, the elevation of serum ALP in G VIII NSBC was highly significant whereas serum bilirubin was not significantly varied. The reverse was observed for G VII heptocellular cancer with cirrhosis. However, it is obvious that the greatest disturbance in liver function occurred in groups that suffered from chronic hepatic disorders including cirrhosis (G III, IV), hepatitis (G IV) and schistosomiasis (G IV, V,IX) whereas the smallest changes were observed in G VII (hepatoma only).

Third, AST/ALT ratio was highly elevated in chronic schistosomiasis but not in active schistosomiasis (G VI), other mixed liver diseases (G II, III, VII) or NSBC (G VIII). It is known that AST is present in a wide variety of tissues including heart, skeletal muscles, kidney, brain and liver whereas ALT appears to be localized primarily in the liver (Boyde and Latner, 1961). In liver, AST is present in both the mitochondria and the cytosol (Boyd, 1961) while ALT is primarily present in the cytosol (over $85 \%$ of total activity) (De Rosa and Robert, 1975). Therefore, the anticipated increase in AST/ALT ratio in chronic schistosomiasis may be ascribed either to a high efflux of AST over time from tissues other than the liver (e.g the heart) or to a slow developing deleterious effect of schistosomiasis on hepatocyte organelles, including mitochondria, causing release of mitochondrial AST into the cytosol, thus increasing AST releasable upon hepatocyte injury (given that the total content of AST in human liver is greater than ALT). A third possible explanation is an effect on the level of pyridoxal-5-phosphate, a cofactor required for the activity of AST and ALT. It has previously been observed that deficiency of pyridoxal-5-phosphate affects hepatic ALT more than AST (Lumeng et al., 1978), leading to an increase in hepatic AST/ALT ratio and a parallel increase in serum AST/ ALT ratio (Ludwig and Kaplowitz, 1980). Thus, AST/ALT represents an interesting issue in the differentiation between SBC and NSBC and between active and chronic schistosomiasis.

Regarding effects on renal function, results indicate that serum urea and creatinine levels did not correlate with each other among the groups. Urea/creatinine ratio was 
elevated in schistosomal patients (G IV, V, VI and IX) while decreased compared to the control ratio in other groups. This could mean that the elevation of serum urea in schistosomiasis is due to induction of urea synthesis in liver rather than an influence on renal excretion function particularly since the change in serum creatinine does not reach twice the average control level. Determination of serum uric acid was unhelpful as almost all data were within the accepted normal range.

Several reports have indicated that schistosoma hematobium causes the elevation of activities of some lysososmal enzymes such as $\beta$-glucuronidase (Abdel-Tawab et al., 1968) and aryl sulphatase (Posey and Morgan, 1977) in the urine of schistosomal patients. Most studies have focused on the assessment of these two groups of enzymes due to their postulated role in the development of SBC (Boyland et al., 1955 and Tsuneo et al., 1984). The origin of the enzymes in the urine of schistosomal patients is uncertain. They may arise from any part of the urinary system injured by the ova spines or from the bacterial infection that usually accompanies the schistosomal infestation. However, our results seem to contradict previous suggestions. No significant increase in NAG activity was observed in the urine of active schistosomal or SBC patients. On the other hand, extremely high NAG activity of was found in the urine of NSBC patients. This surprising observation requires more focused studies in a larger group of patients and this is included in our future plans.

In the last two decades, several studies on plasma a.a have been published to describe the influence of renal disease (McGale et al., 1972) and liver disease (Fischer et al., 1974), on plasma a.a level. Disturbances in the plasma a.a. profile represented by a fall in the serum concentration of branched a.a and elevation of the aromatic a.a were previously recorded in chronic liver disease (Morgan et al., 1982). On the other hand, in acute liver disease, all plasma a.a levels were greatly elevated, except those of branched a.a (Rosen et al., 1977). Hypoaminoacidemia and alteration of plasma a.a pattern was also reported in bladder cancer patients (Nechiporenko et al., 1990). A pathophysiological explanation of these abnormalities implicated imbalance between hepatic clearance and peripheral utilization of the a.a (Soeters et al., 1977).

Despite the presence of variable liver and bladder insults in our patients, the present findings indicate an elevation of the total serum a.a level for all studied groups. The most characteristic observations of this study can be summarized as follows. First, glutamine content was reduced in all patients whereas glutamic acid level was increased. Therefore, glutamine/glutamic acid ratio was remarkably reduced as compared to normal. Second, methionine content was specifically elevated in chronic schistosomiasis. An elevation of serum methionine has previously been observed in chronic hepatic disorders including chronic active hepatitis, primary biliary cirrhosis, cryptogenic cirrhosis and alcoholic hepatitis+cirrhosis (Morgan et al., 1982). Third, SBC patients can be distinguished from the two other types of cancer (NSBC and hepatoma), by an elevation of the serum levels of basic a.a and leucine and reduction of glutamic acid and alanine. Fourth, leucine content was elevated in all schistosomal patients whether active, chronic or associated with bladder cancer. A biochemical basis for these disturbances in serum a.a. level is not immediately obvious. Several factors could be involved, including hepatic synthesis, peripheral utilization, hormone levels and nutritional impact of disease. Furthermore, it is difficult to determine any causal relationship between the a.a disturbance and the disease. 
During the past few years, determination of LDH activity and especially of the LDH isoenzymes has proved useful in the verification of clinical diagnosis of various disorders. The activity of the enzyme in serum is known to be elevated in a number of diseases including myocardial infarction, liver injury and malignant diseases. With regard to malignancy, elevated serum LDH activity has been demonstrated in a variety of carcinomas including liver, breast, bladder, stomach, lung and acute leukemia. In addition serum LDH activity has been shown to correlate with tumor mass in solid tumors (Friedman, 1980). In our study, total serum LDH activity was elevated in all groups except II and V. The significance of total serum LDH in differential diagnosis is generally agreed to be limited. It is rather more useful in the monitoring of patient status. On the other hand, estimation of the isoenzymes pattern in serum may generate different profiles, more or less specific for certain disease states. Our study has demonstrated marked differences in LDH isoenzyme profiles of tested patients.

We conclude that determination of LDH isoenzyme pattern requires further validation in order to be a useful guide or "fingerprint" in the diagnosis, monitoring and treatment of various hepatic and schistosomal disorders.

Overall this study has provided new information on biochemical markers that contribute, alone or in combination, to diagnostic differentiation between mixed hepatic and urinary tract disorders that represent serious health problems in the Egyptian population. Follow-up of these findings should increase our understanding of the pathophysiology of these conditions.

\section{REFERENCES}

Abdel-Tawab, G.A., El-Zoghaby, S.M., Abdel-Samie, Y.M., Kazi, A.M., Kholef, T.S., ElSewedy, S.M. (1968). Urinary $\beta$-glucuronidase enzyme activity in some bilharzial urinary tract diseases. Trans. Roy. Soc. Trop. Med. Hyg., 62, 501-505.

Beaufay, H., Amar-Costesec, A., Feytmans, E., Thines-Sempoux, D., Wibo, M., Robbi, M., Berthet, J. (1974). Analytical study of microsomes and isolated subcellular membranes from rat liver: I. Biochemical methods. J. Cell Biol., 61, 188-200.

Boyd, J.W. (1961). Intracellular distribution latency and electrophoretic mobility of L-glutamateoxaloacetate transaminase of rat liver. Biochem. J., 80, 18(p)-19(p).

Boyde, T.R.C., Latner, A.L. (1961). Starch- gel electrophoresis of transaminases in human tissue extracts and serum. Biochem. J., 82, 51(p)-53(p).

Boyland, E., Wallace, D.M., William, D.C. (1955). Urinary enzymes in bladder cancer. Br. J. Urol., 27, 11-14.

De Rosa, G., Robert, W.S. (1975). Metabolic implications of the alanine aminotransferase isoenzymes. J. Biol. Chem., 250, 7961-7967.

Fawcett, J.K., Scott, J.E. (1960). A rapid and precise method for the determination of urea. J. Clin. Path., 13, 156-159.

Fischer, J.E., Yoshimura, N., Aguirre, A., James, J.H., Cummings, M.C., Deindoerfer, F. (1974). Plasma amino acids in patients with hepatic encephalopathy: effects of amino acid infusions. J. Surg., 127, 40-47.

Friedman, R.B. (1980). Effect of diseases on clinical laboratory tests. Clin. Chem., 26 (suppl. 4), 92D.

Hamilton, P.B. (1962). Ion exchange chromatography of amino acids. Ann. N.Y. Acad. Sci., 102, $55-62$.

Henny, J., Schiele, F., Young, D.S. (1985). In: Siests (Ed). Interpretation of Clinical Laboratory Tests. Biomedical Publication, 220-234. 
Huang, H.J.S., Yee, J.K., Shew, J.H., Phang-Lang Chen, Robert, B., Theodore Freidmann, Eva, Y.H.P. Lee, Wen-Hwa Lee (1988). Suppression of the neoplastic phenotype by replacement of the R.B. gene in human cancer cells. Science, 242, 1563-1566.

Jendrassik, L., Grof, P. (1938). Vereinfachte photometrische Methoden zur Besimmung des Blutbilirubins. Biochem. Zeit., 297, 81.

Lowry, O.H., Rosebrough, N.J., Farr, A.L., Randall, R.J. (1951). Protein measurement with Folin phenol reagent. J. Biol. Chem., 193, 265-275.

Ludwig, S., Kaplowitz, N. (1980). Effect of pyridoxine deficiency on serum and liver injury in the rat. Gastroenterology, 79 (3), 545-549.

Lumeng, L., Ryan, M.P., Li, T.K. (1978). Validation of the diagnostic value of plasma pyridoxal 5-phosphate measurements in vitamin B6 nutrition. J. Nutr., 108 (4), 545-549.

Mahmoud, A.F., Abdel Wahab, M.F. (1990). Trematodal infections. In: Tropical and Geographical Medicine, second edition, 458-479.

McGale, E.H.F., Pickford, J.C., Aber, G.M. (1972). Quantitative changes in plasma amino acids in patients with renal disease. Clin. Chim. Acta, 39, 395-403.

Michael, C.K. (1990). Hepatic tumors. Medicine Intern., 48, 3492-3501.

Morgan, M.Y., Marshall, A.W., Milsom, J.P., Sherlock, S. (1982). Plasma amino-acid patterns in liver disease. Gut, 23, 362-370.

Nechiporenko , N.A., Nefedov, L.I., Klimovich, I.I. (1990). The free amino acid content of the blood serum in bladder cancer patients. Urol. Nefrol., 5, 17-20.

Pohl, A.L. (1992). How to select and rate clinically relevant tumor markers. Egy. J. Tumor Marker Onc., 3, 1-9.

Posey, L.E., Morgan, L.R. (1977). Urine enzyme activities in patients with transitional cell carcinoma of the bladder. Clin. Chem. Acta, 74 (1), 7-10.

Ragheb, M. (1956). Schistosomiasis of the liver: clinical, pathological and laboratory studies in Egyptian cases. Gastroenterology, 30, 631-660.

Rosen, H.M., Yoshimura, N., Hodgman, J.M., Fischer, J.E. (1977). Plasma amino acid patterns in hepatic encephalopathy of differing etiology. Gastroenterology, 72, 483-487.

Soeters, P.B., Weir, G., Ebeid, A.M., Fishcer, J.E. (1976). Insulin, glucagon, portal systemic shunting and hepatic failure in the dog. J. Surg. Res., 23 (3), 183-188.

Tietz, N.W., Rinker, A.D. (1983). The measurement of alkaline phosphatase (ALP). In: Homberger (Ed). Analytical Concepts in Enzymology: Skokie, IL. College of American Pathologists, 195203.

Tsuneo, O., Mikio, K., Kazuo, O., Kikuko, I. (1984). Urinary enzyme determination and its clinical significance. Biochem. Med., 32 (3), 349-356.

Wacker, W.E.C., Ulmer, D.D., Vallee, B.L. (1956). Metaloenzymes and myocardial infarction (II) malic and lactic dehydrogenase activities and zinc concentration in serum. New Eng. $J$. Med., 255, 499-511.

Watts, R.W.E. (1974). Determination of uric acid in blood and urine. Ann. Clin. Biochem., 11, 103-111.

Wilkinson, J.H. (1976). Principles and Practice for Diagnosis Enzymology. Second ed. Philadelphia. J.B. Lippincott Co., 282-319.

Wroblewski, F., La Due, J.S. (1956). Serum glutamic-pyruvic transaminase (SGPT) in hepatitis disease: A preliminary report. Proc. Soc. Exp. Biol. Med., 91, 801-811. 


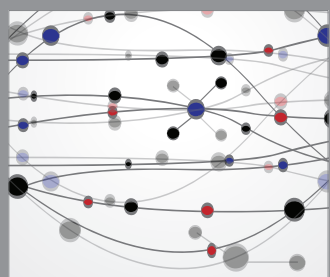

The Scientific World Journal
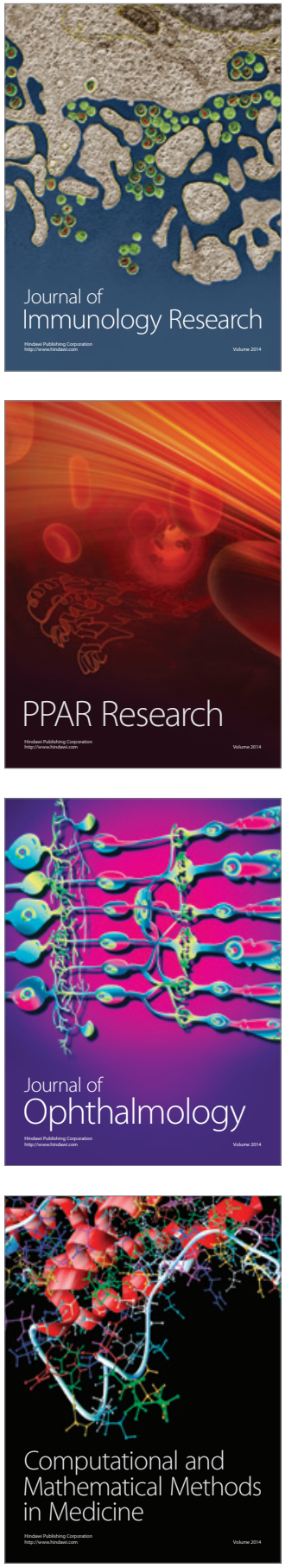

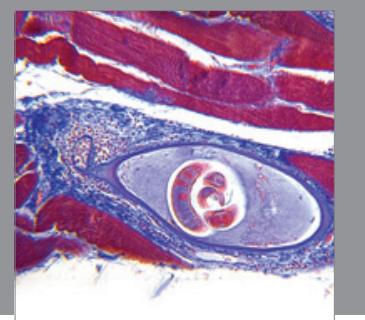

Gastroenterology

Research and Practice
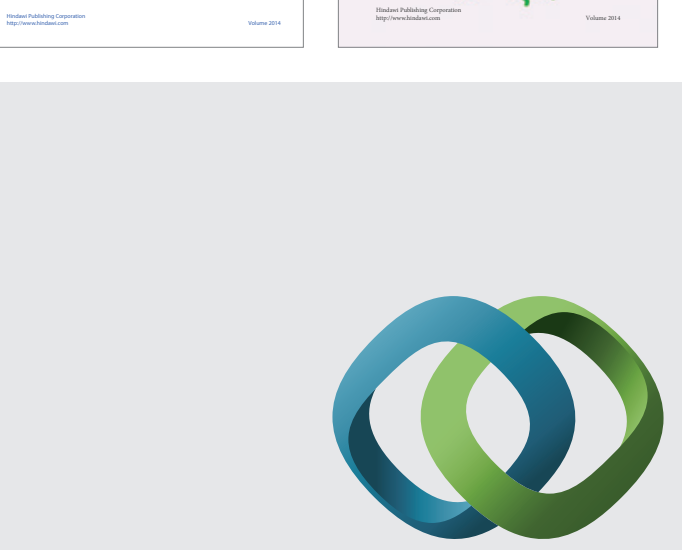

\section{Hindawi}

Submit your manuscripts at

http://www.hindawi.com
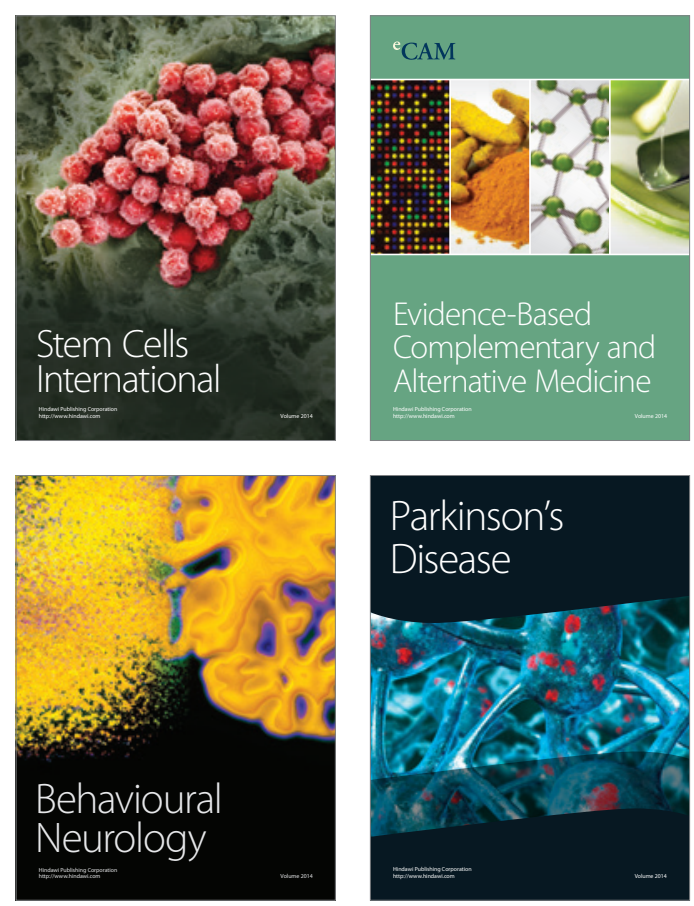

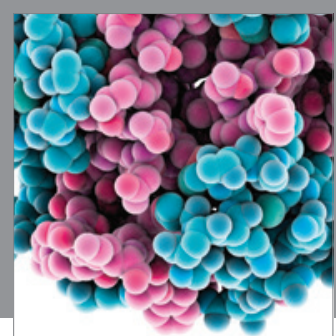

Journal of
Diabetes Research

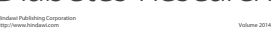

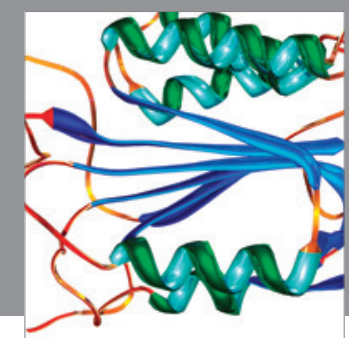

Disease Markers
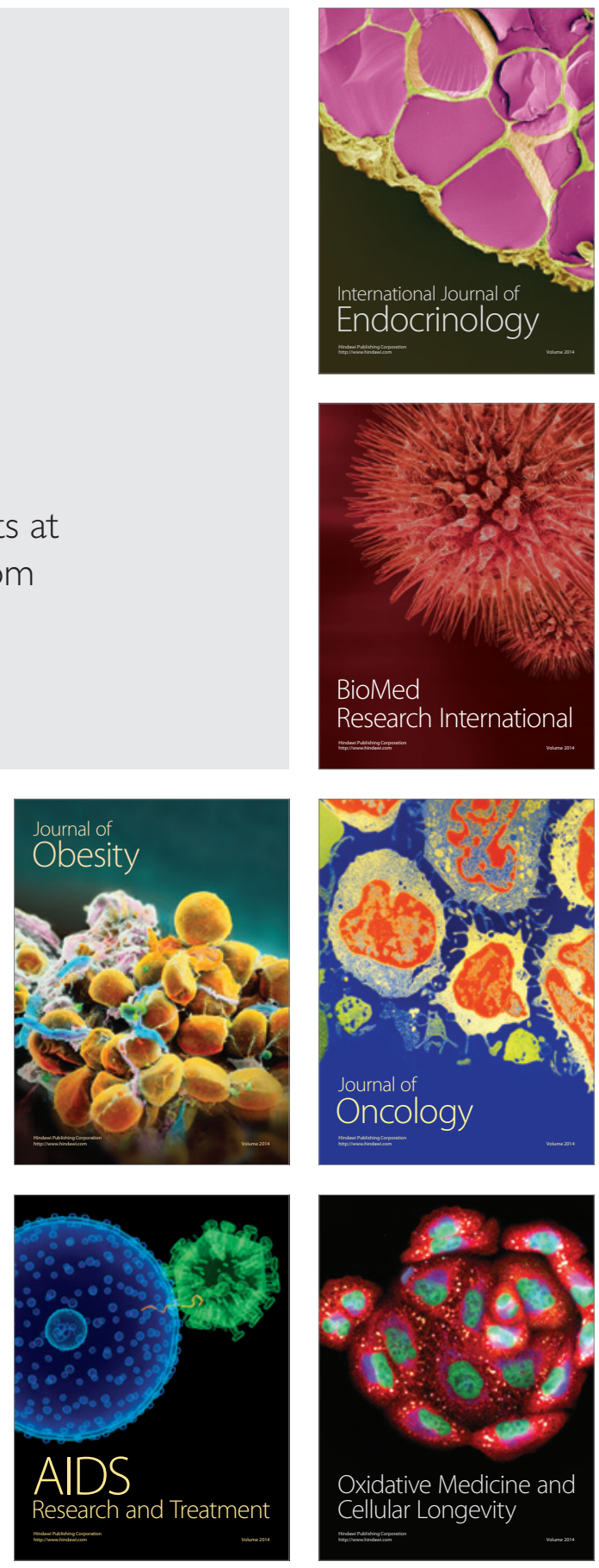